\title{
Osteochondral Integrated Scaffolds with Gradient Structure by 3D Printing Forming
}

\author{
Wei-Hua Chen Yuan-Yuan Liu Fu-Hua Zhang Yong-Ze Yu Hai-Ping Chen Qing-Xi Hu \\ Rapid Manufacturing Engineering Center, Shanghai University, Shanghai 200444, China
}

\begin{abstract}
Recently in the area of biological manufacturing and rapid prototyping manufacturing, the bone scaffolds based on the additive manufacturing in repairing bone defects have been paid more and more attention. In the process of preparation, path planning directly affects the structure, performance as well as the final bone cell culture conditions. Due to the special natural bone scaffold structural characteristic, the traditional rapid prototyping (RP) path planning is not fully suitable for the preparation of bone scaffolds. In this paper, based on the 3D printing extrusion forming technology, a method of path planning for osteochondral integrated scaffolds with gradient structure is put forward, which provides a theoretical basis for bone-scaffold modeling and practical preparation. The implementation of the path planning processing system makes it possible to process data automatically from the initial stereo lithography (STL) model of the actual bone defect part by computer X-ray tomography technique (CT) scan or modeling, to generate the path code and to generate the final machining information after post-processing. This work provides some guidelines for independent research and development of automation equipment for biological manufacturing preparation and software technology. The experiment and test results have verified the validity of the path planning method and the good properties of the bone scaffolds with gradient structures.
\end{abstract}

Keywords: 3D printing, biological bone-scaffold, path planning, additive manufacturing, tissue engineering.

\section{Introduction}

The human joint tissue defect or injury caused by sports injuries, accidents, natural disasters and other causes is very common in clinical practice, which has become one of the main causes of physical disabilities. The articular osteochondral injury is often accompanied by joint mechanical stress changes. If not promptly treated, it will further lead to osteoarthritis, seriously affecting people's life quality. Due to the special physiological structure of joint tissue, the repair ability of human body joint articular osteochondral tissue is poor. The traditional method of bone cartilage tissue repair is difficult to obtain a satisfactory repair effect. Therefore, using tissue engineering technique to repair osteochondral defects has become the current focus of research at home and abroad. The human joint tissue defect or injury caused by sports injuries, accidents, natural disasters and other causes is very common in clinical practice, which has become one of the main causes of physical disabilities. The articular osteochondral injury is often accompanied by joints mechanical stress changes, if not promptly treated, it will further lead to osteoarthritis, seriously affecting people's life quality. Due to the special physiological structure of joint tissue, the repair ability of human body joint articular osteochondral tissue is poor. The traditional method of bone cartilage tissue repair is

\footnotetext{
Regular paper

Manuscript received September 29, 2013; accepted March 28, 2014

This work was supported by National Natural Science Foundation of China (Nos. 51475281 and 51375292), National Youth Foundation of China (No. 51105239)

Recommended by Guest Editor Xin Sun

(C)Institute of Automation, Chinese Academy of Science and Springer-Verlag Berlin Heidelberg 2015
}

difficult to obtain a satisfactory repair effect, therefore, using tissue engineering technique to repair osteochondral defects has become the current focus of research at home and abroad $^{[1]}$. However, due to the limitations of process and equipment technology, the current preparation of the bone cartilage often lacks the transition layer between the bone and cartilage, which limits the integration forming. Thus, the repaired tissue in dispersing stress, resistance to shear and the free tissue fluid exchange limit between the bone and cartilage tissue cannot meet the clinical need.

The 3D printing forming technology with multi-type nozzles working coordinately can form the osteochondral integration scaffolds once-through, consisting of osteochondral layer, bone layer and transition layer with gradient material distribution and internal gradient pore structure, which could effectively repair the osteochondral tissue defects and provide a new train of thought and method ${ }^{[2-4]}$.

Currently, on the one hand, when forming the initial defect model by scan rebuild or design as input, the studies on the preparation of biological bone scaffold are mainly focused on the experimental stage of process improvement and material testing. There are few from this point of view that equipment how to generate the processing information needed to drive the hardware according to a certain path for the bone scaffold structure. Also, there is not a general set of path planning algorithms applicable to the 3D printing bone scaffold process. On the other hand, a large number of domestic and international research works are mainly focused on the method to reconstruct the 3D shape of human organs or tissue engineering scaffold structure modeling ${ }^{[5]}$. Because there is no from the viewpoint of the process and 
the feasibility of preparation, few model designs are finally prepared. Therefore, oriented in the feasibility of biological bone scaffold preparation, the proposal of the path planning algorithm with a certain generality has a strong practical meaning for the present modeling and preparation.

The rest of the paper is organized as follows. Section 2 describes the scaffold forming process by $3 \mathrm{D}$ printing technology and the analysis of the scaffold structure. According to the structure features, two types of path lines are introduced. Section 3 introduces the detail path planning process and algorithm for functional gradient scaffolds. Section 4 introduces the implementation of the path planning software system and displays the friendly human-interface. The experimental method is given and the results are analyzed in different aspects in Section 5. Section 6 concludes the paper.

\section{Path planning for 3D printing bone scaffolds}

\subsection{D printing forming bone scaffolds technology}

Compared with the traditional scaffold forming method, $3 \mathrm{D}$ printing technology has unique advantages in manufacturing complex three-dimensional structures of bone scaffold. The forming process is shown in Fig. 1.

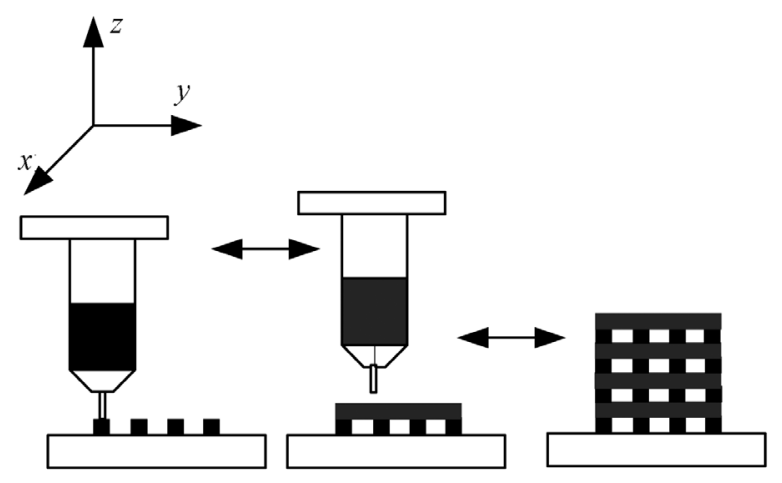

Fig. $13 \mathrm{D}$ printing forming process

At an appropriate temperature and pressure condition, firstly, the receiving platform moves to the bottom of feeding nozzle location. Then the bone scaffold material is continuously extruded and solidifying with the receiving platform moving regularly in the $X$ and $Y$ directions according to the desired macro-pore. When completing one layer of bone scaffolds, then the nozzle device rises to a certain height with the platform moving in the other direction to form the next layer. And this cycle goes on back and forth, ultimately the biological bone scaffolds with a three-dimensional structure is prepared.

The technology has the advantages such as high manufacturing accuracy, short manufacturing cycle and strong manufacturing repeatability. However, due to the lack of path planning, the technology is now mainly used to pre- pare scaffolds with regular shape and pore structures, as shown in Fig. 2. There are still some limitations for the gradient changes in material and pore structure of the osteochondral scaffolds.

\subsection{Path planning for 3D printing scaffolds}

A series of path planning algorithms have been put forward based on the traditional rapid prototyping process, such as parallel line scanning methods, contour offset scanning ways, spiral scanning path, the path based the Voronoi diagrams, and so on, or based on a combination of two or more of the above scan path algorithms. All these algorithms are mainly aimed at rapid prototyping process defects like buckling and deformation, which also can reduce the idle motion, improve efficiency and improve surface finish, etc. ${ }^{[6,7]}$

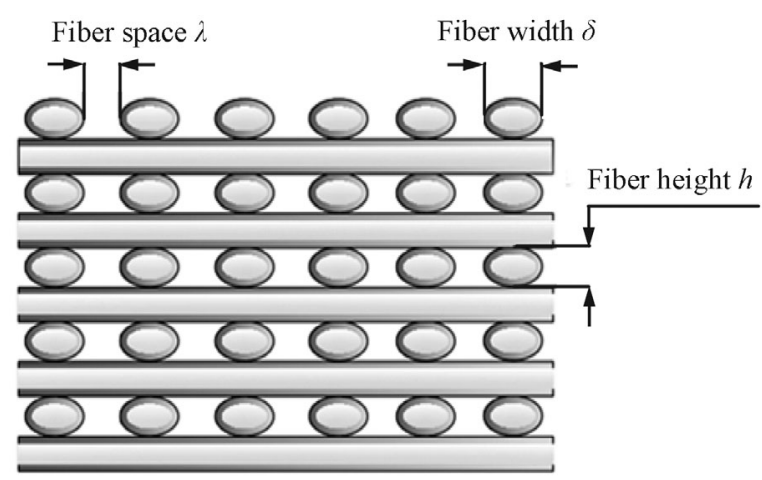

Fig. 2 Cross section of three-dimensional scaffolds structure

For bone scaffold, the $3 \mathrm{D}$ printing scan path is the very fiber forming position. In the traditional rapid prototyping process, adjacent fibers fit closely. However, in order to ensure the through pores in the three-dimensional bone scaffold, adjacent forming fibers must leave the space. Besides the type I scan line in both paths, there is also a unique type II scan line in bone scaffold path called the transition scanning lines, as shown in Fig. 3. The transition lines have an impact on scaffold forming because it is at this area where some process problems such as jetting or material over-accumulation may happen, thus affecting the overall quality and shape accuracy of the scaffolds ${ }^{[8-10]}$.

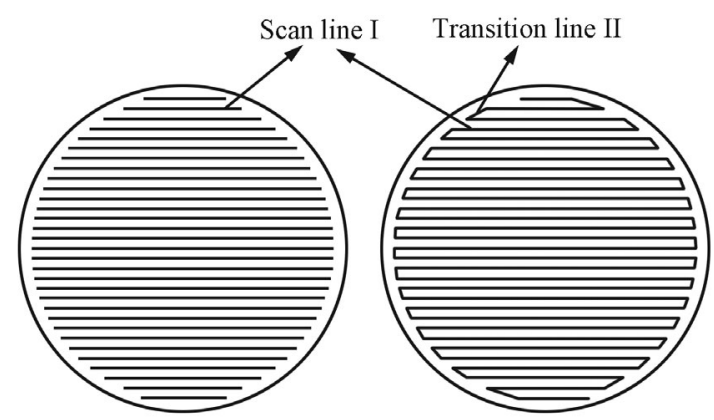

Fig. 3 Types of scan lines in bone scaffold

From the perspective of the characteristics of biological bone scaffolds structure and combining the existing process, 
the parallel scanning way is the most appropriate to reduce the complexity of the process and meet the requirements of scaffolds at the same time. It can improve the efficiency and reduce the frequent start and stop of the nozzle feeding device to ensure the effect and quality to a certain extent. In addition, although the contour offset path planning can get contour approximation to achieve overall dimensions, for the late cell culture stage, the scaffolds must have sufficient side pores to ensure the exchange of nutrients and metabolites, so that the contour offset scanning path is not adopted.

\section{Realization of functional gradient scaffolds}

\subsection{Structure characteristics}

The natural articular cartilage structure has a multigradient structure, including transparent cartilage layer, osteochondral transition layer and subchondral bone layer. Each part is obviously different in composition, structure and cellular components than others. The particular osteochondral physicochemical properties and gradient characteristics require a specific material gradient and gradient structure to achieve the functional and effective alternatives.

At present, the single material and uniform pore structure of the prepared scaffold will affect cell growth behavior, and even the tissue function of the repair in vivo. In addition, the existing technology to build the osteochondral scaffolds lacks the structure of the interface between bone and cartilage layer, named the bone and cartilage transition layer. The transition layer plays an important part in support, isolation, mechanical coupling stress, buffering stress and maintaining cellular microenvironment and other physiological functions.

\subsection{Path planning for bone scaffold gradi- ent structure}

There are two main sources for bone defect model, one is through computer X-ray tomography technique (CT) scan and $3 \mathrm{D}$ reconstruction, the other is through direct model design, usually the stereo lithography (STL) model as input. By slicing the STL model, the contour data of each layer could be obtained and stored for the path planning algorithm to process.

To form the integrated functionally gradient osteochondral scaffold, the first key is to form an internal gradient structure in the scaffold. The parallel scan path planning is used to form different gap spacings on demand, after layer by layer to eventually form the gradient structure scaffold. The basic steps of the path generation algorithm are as follows $^{[11]}$ :

1) Contour circle building through STL-sliced data,

2) Determination of unit scan line,

3) Scanning lines intersecting,

4) Selection and sorting for intersection point constitut- ing gradient scan path,

5) Generating the node pairs,

6) Storing the scan lines.

Fig. 4 shows the contour circle building through the data after the slicing process of the STL model, where points $P_{A}, P_{B}, P_{C}, P_{D}, P_{E}$ and $P_{F}$ constitute a polygon. Then from the desired scanning direction the base scan lines 1 , $2,3, \cdots$, and the starting scan line, and init_scanline are determined, with $\lambda$ as the smallest unit of parallel scan line spacing.

$$
\text { line }_{\text {between }}=\lambda \text {. }
$$

The intersection points are found by the line segment of adjacent points and determined scan lines. Taking line segment $P_{A} P_{B}$ as example, the limit scan lines that can intersect the line segment are

$$
\begin{aligned}
& \text { scanline }_{\text {start }}=\operatorname{int}\left(\frac{P_{A}[y]-\text { init }_{\text {scanline }}}{\text { line }_{\text {between }}}\right) \\
& \text { scanline }_{\text {end }}=\operatorname{int}\left(\frac{P_{B}[y]-\text { init }_{\text {scanline }}}{\text { line }_{\text {between }}}\right) .
\end{aligned}
$$

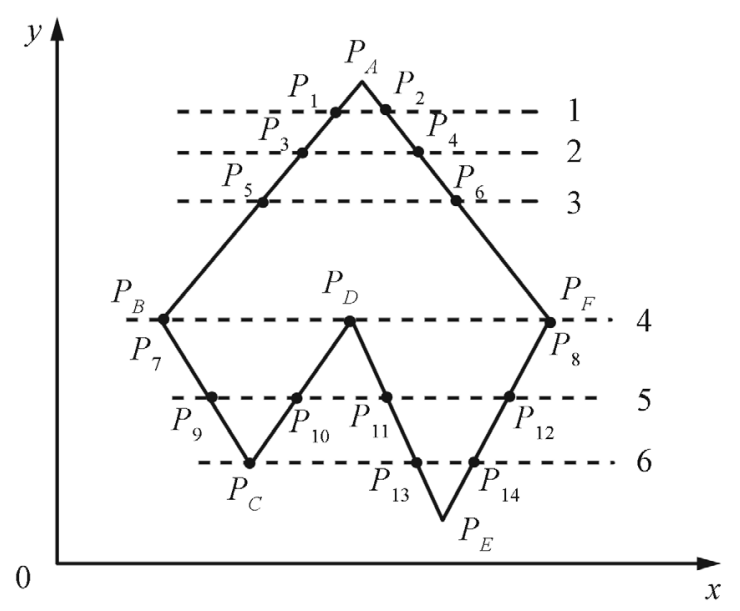

Fig. 4 Schematic parallel scanning path generating method

To do the intersection calculation, the coordinates of two points $P_{A}$ and $P_{B}$ are set to $\left(x_{1}, y_{1}\right),\left(x_{2}, y_{2}\right)$, where $x_{1}<$ $x_{2}$. When the scanning line and line segment $P_{A} P_{B}$ are overlapped, the intersection of these two lines are recorded as $\left(x_{1}, y_{1}\right)$, otherwise denoted as $\left(x_{0}, y_{0}\right)$, and

$$
x_{0}=\frac{\left(x_{2}-x_{1}\right) \times\left(y_{0}-y_{1}\right)}{\left(y_{2}-y_{1}\right)}+x_{1} .
$$

Go on finding the next adjacent line segment intersection point after finding one segment intersection until all intersection points by segments and scan lines are found.

The scanning intersection points are obtained by the scanning lines with $\lambda$ as the minimum unit. Then according to the requirements of the scaffold structure, operators can choose different scanning line spaces for different layers. And through the scanning line spacing increasing or decreasing between layers, the gradient structure is formed. The final step is to match the scanning intersection points, starting from the first point, to find the corresponding end 
of the scanning line so that forming the scanning line I. According to the reciprocating parallel scanning way in a certain order, the scanning line II is formed connecting the end points of the former line I and the start points of the next line I on the same side. After the procedure is repeated, eventually the Zig-zag path information is generated. Fig. 5 shows the three-dimensional structure with three different fiber gaps form the top to the bottom, in which the centerto-center spacings between the fibers are respectively $4 \lambda$, $5 \lambda$ and $6 \lambda$. Thereby a gradient structure between different layers of the scaffold is formed. Meanwhile, according to different needs, the fiber gap changes may be also different within the same layer so as to form sparse and dense gradient changing structures, as shown in Figs. 6 (a) and (b).

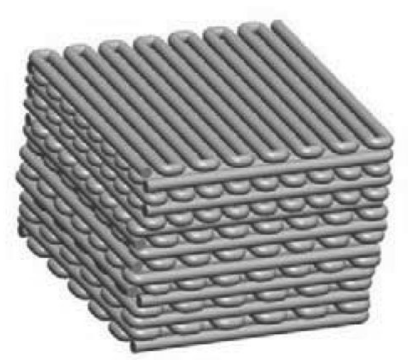

(a)

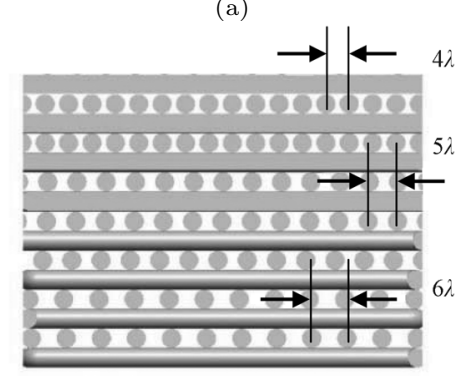

(b)

Fig. 5 Gradient structure between layers in bone scaffold

With different angles, the overlay of scanning paths in layers could produce different pore structures, as shown in Fig. 7 , which also has a certain influence on the mechanical properties and affect the cell adhesion and survival growth state at a post cell culture stage to some extent ${ }^{[12]}$.

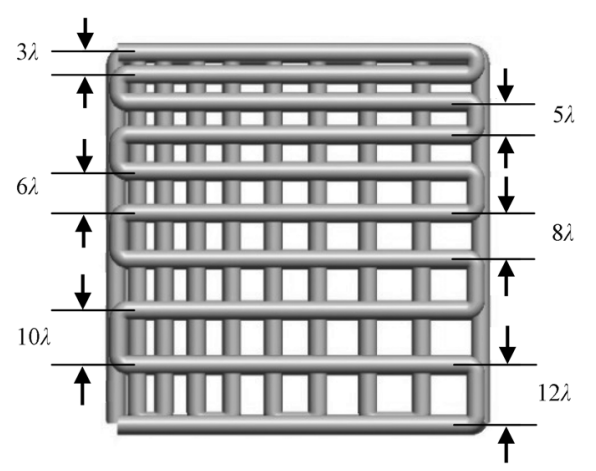

(a)

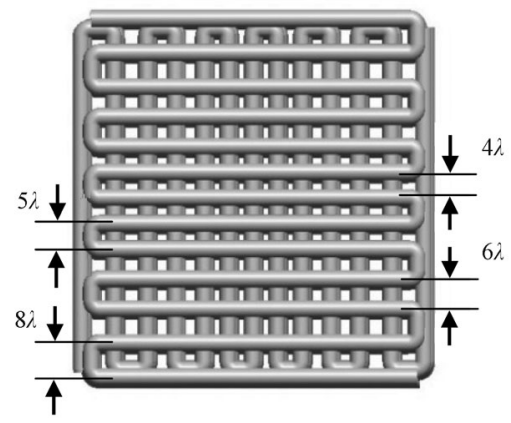

(b)

Fig. 6 Gradient structure within one layer and between layers in scaffold

\subsection{Realization of material-gradient scaf- folds}

From the viewpoint of this thesis, the gradient structure formed by path planning is a basis for the formation of material gradient scaffold. On this basis, there are two ways to form a material gradient scaffold: 1) With the gradient structure changes in scaffold, selecting different feeding nozzles with different materials or compositions to form layers with different fiber gaps, in order to achieve different structural layers of different materials to realize the materialgradient scaffolds. 2) Under the condition of the same structure, adding the parameters of the switch nozzle control as flags into the path planning processing information, so that the scanning path can include the parameters of selected nozzle with specific material. In this way, different path could be formed with different materials by detecting the nozzle flags in the path planning information to achieve the material-gradient scaffold.

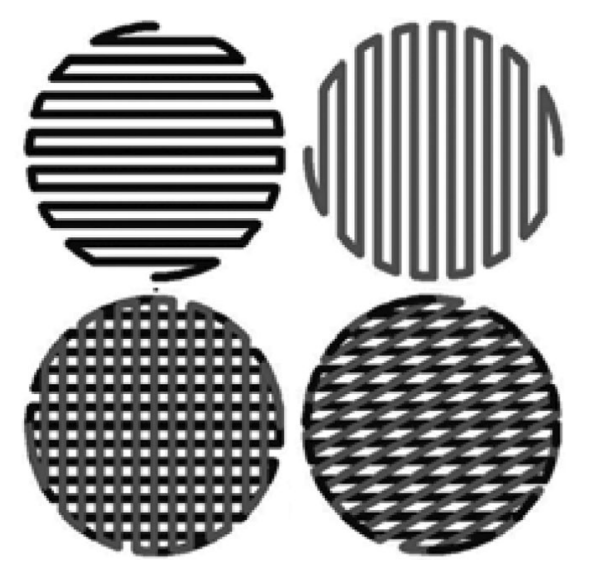

Fig. 7 Schematic of overlapped scanning paths with different angles

Also the two ways above can be combined for a more complex and flexible material-gradient scaffold design and preparation. As the processing information could drive the underlying hardware devices after post-processing, the design or the modification of the path information could make the scaffolds preparation more controllable and simpler, improving the reproducibility and quality of the scaffold and 


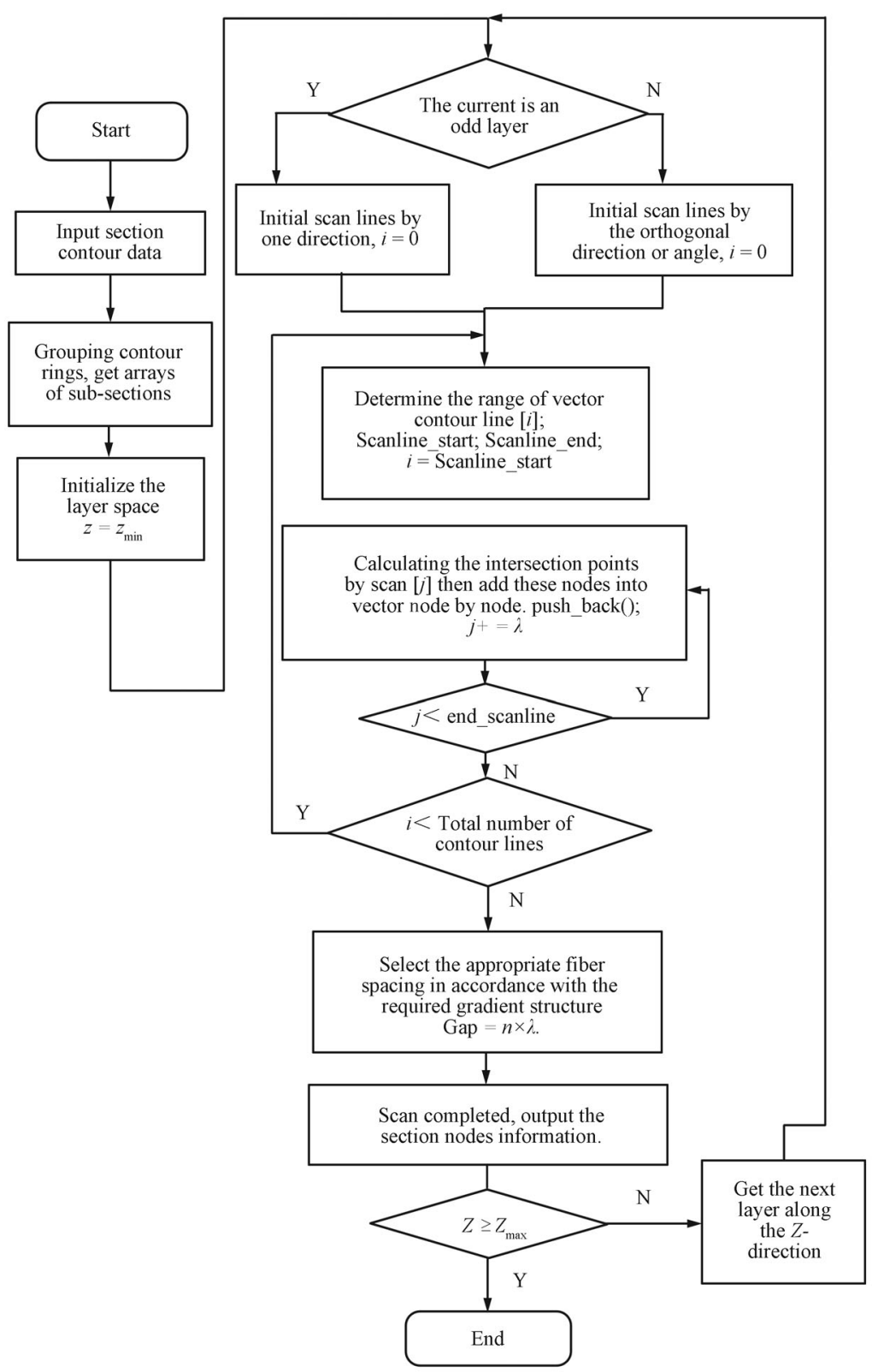

Fig. 8 Algorithm flowchart of bone scaffold path generation with gradient structure
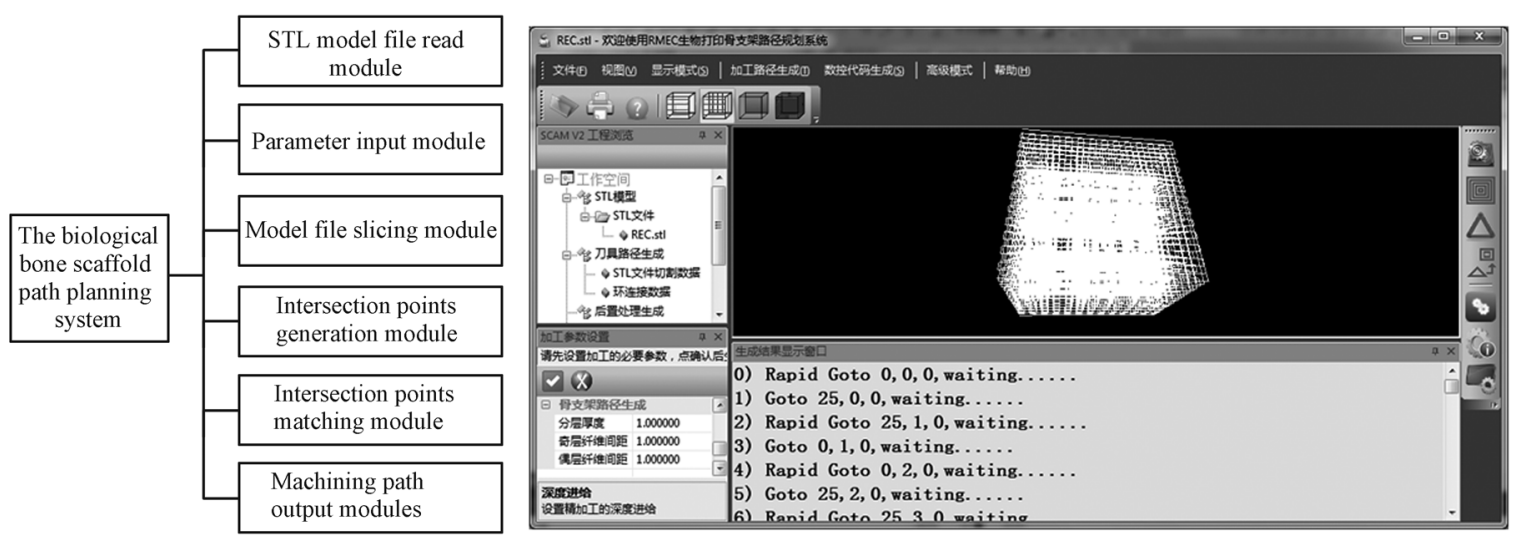

Fig. 9 The bone scaffold path planning system 
greatly reducing the human factors on the final effect during the preparation. In summary, the flow chart of the path scanning algorithm with gradient structure of bone is shown in Fig. 8.

\section{Biological bone scaffold path plan- ning system implementation}

According to the path planning algorithm research for gradient scaffold in this study, a biological bone scaffold path planning system was developed using $\mathrm{C}++^{[13]}$. There are six major modules, as shown in Fig. 9: STL model file read module, parameters input module, model file slicing module, intersection points generation module, intersection points matching module and machining path output module. The software interface consists of the parameters input part, the model dynamic display part and the generated results display part. After the STL input is processed by the software system, the information is obtained for driving the device to operate ${ }^{[14]}$.

\section{Experimental results}

\subsection{Experimental method}

The gelatin was dissolved in deionized water to prepare the $20 \%$ transparent solution, the sodium alginate powder was dissolved in deionized water to prepare a $3.5 \%$ milky white solution, which was then mixed with the same quality of hydroxyapatite (HA) and water to form suspension. In a water bath at $60^{\circ} \mathrm{C}$ environment, it was mixed and stirred with a $20 \%$ gelatin solution thoroughly to form the component material for one nozzle. Chitosan was dissolved in a $1 \%$ acetic acid solution to prepare the $5 \%$ chitosan solution at a temperature of $60^{\circ} \mathrm{C}$ environment. Then, we mixed the $5 \%$ chitosan solution and $20 \%$ gelatin solution to prepare the gelatin-chitosan solution with the mixture ratio of $1: 1$, and the mixed solution was used as a component material in the feeding nozzle.

The experimental equipment is a self-developed 3D biological printing platform. The control unit of the platform is the Delta Tau's programmable multi-axes controller (PMAC), and stepper motors and multi nozzles are the execution unit working collaboratively controlled by PMAC. The desired shape of the scaffold is inputted to the STL file, through path planning system processing, the desired scanning path and machining information are obtained (gradient or non-gradient structure), then after the postprocessing system, we are finally capable of driving the hardware to print the scaffolds.

\subsection{Experimental results and analysis}

\subsubsection{Macro-morphology of the scaffolds}

Fig. 10 is the gradient structure scaffold with the same material which has not yet been cross-linked and has been freeze-dried for 48 hours. The results from Fig. 10 (a) shows a relatively neat contour and through-pores while Fig. 10 (b) shows the fine through-pores from a side sectional view. From top down, the pore size is changing from large to small, achieving a gradient structure as well as tight adhesion between the layers. Fig. 11 is the micro scanning electron microscopy (SEM) of the scaffolds with gradient structure, it shows that between the adjacent fibers the space is different from layer to layer. The adjacent pore spaces in this picture are respectively $353 \mu \mathrm{m}$ and $529 \mu \mathrm{m}$.

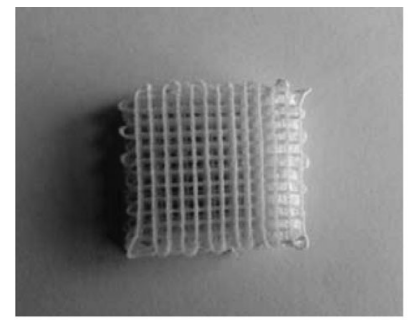

(a)

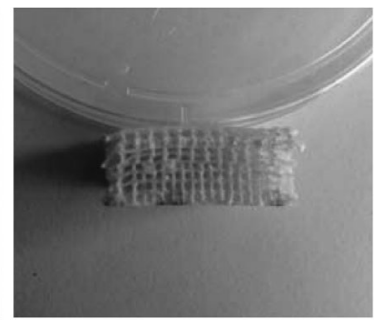

(b)
Fig. 10 Prepared scaffolds with gradient-structure

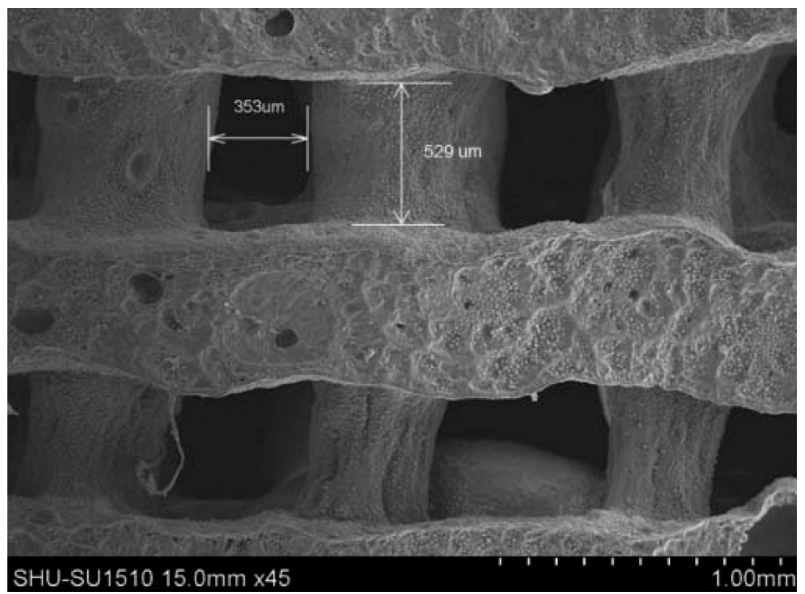

Fig. 11 SEM of the scaffolds with gradient

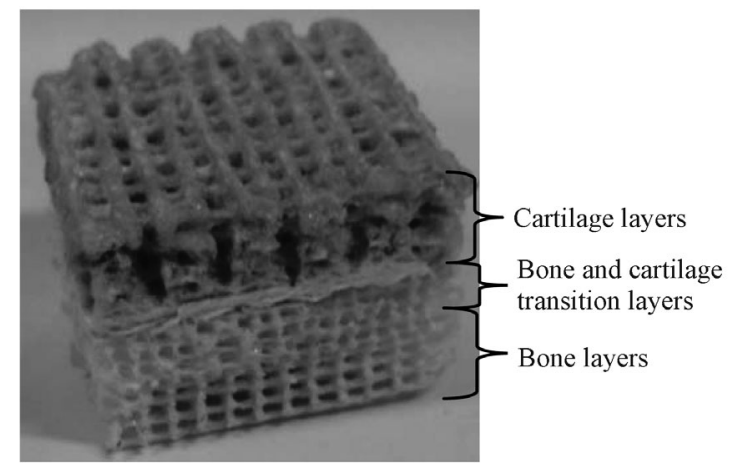

Fig. 12 Shape and structure of the prepared osteochondral integrated scaffold

Fig. 12 shows a functionally gradient osteochondral integrated scaffold which was preliminarily prepared with different gradient structures in different gradient bio-materials using different feeding nozzles. The macroscopic and microscopic observations show that the scaffold has internal connectivity of pore structures with good adhesion to each 
layer. The average pore size of the bone layers is about 250 $\mu \mathrm{m}$, it is about $100 \mu \mathrm{m}$ for the bone and cartilage transition layers and around $300 \mu \mathrm{m}$ for the cartilage layers.

\subsubsection{Mechanical properties}

The mechanical property is also a main indicator to evaluate the pros and cons of the scaffold. In this dissertation, the same size test samples were made from the prepared scaffolds with gradient and non-gradient structures after the treatment of cross-linking and freeze-drying. They were tested for compression mechanical properties through loading on the vertical surface to verity the performance on mechanical properties between functional gradient scaffolds and regular structure scaffolds. From the experimental result in Fig. 13, at the beginning of the loading with displacement and deformation in a small area, there was almost no difference of the receiving force between the gradient and non-gradient structures. With continually increasing the loading of compression, the gradient structure scaffold displayed a larger deformation at first. Under a $5 \mathrm{~N}$ loading, the difference between the two samples reached the maximum and the non-gradient structure scaffold showed a stronger compression resistance performance.

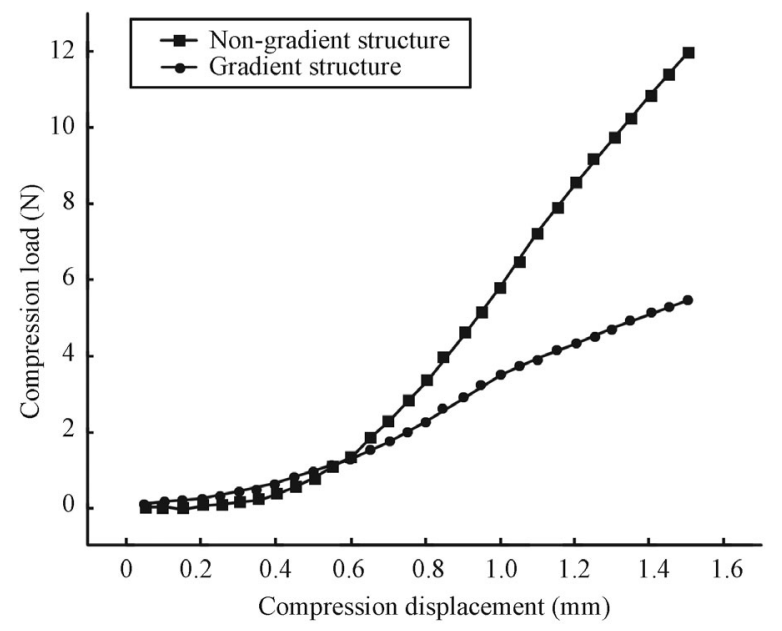

Fig. 13 The compression load-displacement testing

Meanwhile, after elastic modulus test on these two samples as shown in Table 1, the Young's elastic modulus of the gradient structure scaffold was greater, even reached twice more than the non-gradient structure scaffold's. It also showed a certain kind of flexibility, plasticity and ductility. Possessing compression resistance performance is a necessary condition for the scaffold, but at the same time it also needs a kind of plasticity and toughness to make the scaffold more suitable for cultivating in vivo and can relieve the force better from the fluid in a micro-environment, as well as the shearing strain or other forces.

A scaffold should be with suitable mechanical properties such as strength, hardness, toughness and plasticity to satisfy the medical requirement for wearing resistance, pressure durability, impact resistance, fatigue, bending and so on. Therefore, the compression strength and the elastic modulus of a scaffold should combine in a balance so as to show the best performance on the mechanical property. The test demonstrated that no matter for the gradient structure or non-gradient structure scaffold, the compression strength and the elastic modulus were in an acceptable range.

\subsubsection{Cells cultivation}

The adhesion, proliferation, growth and differentiation of the cells on the scaffold are important indicators for the scaffold to be cultivated in vivo. Fig. 14 shows the situation of cell growth on the scaffold surface observed by SEM. In Fig. 14 (a), the white spherical granules in the white circle are cells. A lot of cells could be observed over the surface of the scaffold, but the spherical white granular cells were not growing and adhering to the surface of the fibers. In Fig. 14 (b), tentacles of the cells could be found. The cells extended out and became flat, indicating that cells began to grow adherently to the surfaces.

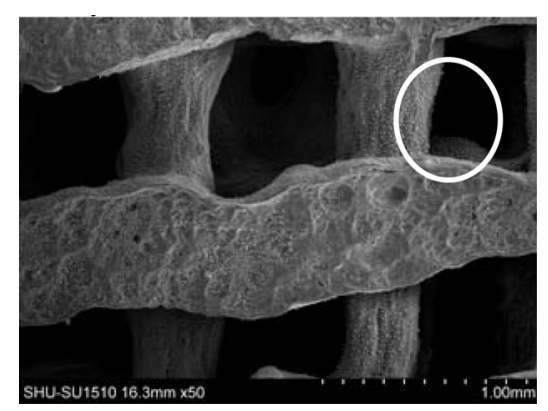

(a)

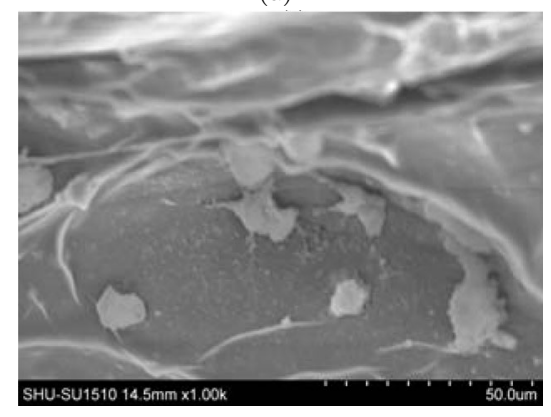

(b)

Fig. 14 The situation of cell growth on the scaffold surface under the SEM

\section{Conclusions}

This dissertation is for the $3 \mathrm{D}$ printing process for preparing biological bone scaffolds, and focuses on the special characteristics of osteochondral integrated scaffolds with gradient structure. Combined with the path scanning algorithm for traditional rapid printing prototyping process, the author makes an applicable biological bone scaffold path planning method. The aim is to produce scaffold with complex contour and gradient structure. Meanwhile, from the 
Table 1 The elasticity modulus test

\begin{tabular}{cccc}
\hline Type of the scaffold & Elastic modulus (Young's) $(\mathrm{MPa})$ & Maximum compression load $(\mathrm{N})$ & Elastic modulus (auto) (MPa) \\
\hline Non-gradient structure & 70.1212 & -124.2965 & 58.03313 \\
Gradient structure & 183.33717 & -80.06853 & 114.13295 \\
\hline
\end{tabular}

perspective of the development of the equipment, we combined process features and considered process parameters, and eventually the hardware platform was driven to print the scaffolds. The scaffolds were prepared with the independent research and development $(\mathrm{R} \& \mathrm{D})$ platform after the STL model was inputted. The followings were verified: 1) The contour of the scaffold prepared was as good as the input model. The macro-pores structures from the vertical and side could meet the requirements of the tissue engineering scaffolds. It realized the structural gradient and functional gradient and confirmed the validity of the algorithm. 2) By the mechanical properties testing, it is demonstrated that no matter for the gradient structure or non-gradient structure, the compression strength and the elastic modulus of the scaffolds should be in an acceptable range. 3) By the cell inoculation experiments, it is shown that the cells could grow adherently to the surfaces of the fibers in the scaffold with good growth and differentiation. 4) The development of the software processing system realized the function as a bridge linking the STL files obtained by CT scanning or modeling of the tissue engineering scaffolds and the actual preparation on the underlying equipment. This provided a theoretical basis for the automation of the biological manufacturing equipment development, and it is of great significance for the repeated and stable preparation of the biological scaffold according to the macro structures required, which will finally meet the increasing experimental and clinical needs in the medical and tissue engineering fields.

This paper mainly focuses on the situation where the section of the STL model is a simple connected area, which means no hollows in the scaffold structure. And only one path planning method, reciprocating parallel path lines, is applied in forming the scaffold. So the future work is to improve the path planning algorithm for scaffolds with hollows inside and to mix other different path planning methods like contour offsetting method to form more complex inner pore structures of the scaffold by 3D printing method.

\section{References}

[1] D. W. Hutmacher. Scaffolds in tissue engineering bone and cartilage. Biomaterials, vol. 21, no. 24, pp. 2529-2543, 2000.

[2] J. M. Sobral, S. G. Caridade, R. A. Sousa, J. F. Mano, R. L. Reis. Three-dimensional plotted scaffolds with controlled pore size gradients: Effect of scaffold geometry on mechanical performance and cell seeding efficiency. Acta Biomaterialia, vol. 7, no. 3, pp. 1009-1018, 2011.

[3] S. A. Park, S. H. Lee, W. D. Kim. Fabrication of porous polycaprolactone/hydroxyapatite (PCL/HA) blend scaffolds using a $3 \mathrm{D}$ plotting system for bone tissue engineer- ing. Bioprocess and Biosystems Engineering, vol. 34, no. 4, pp. 505-513, 2011.

[4] A. A. Sawyer, S. J. Song, E. Susanto, P. Chuan, C. X. F. Lam, M. A. Woodruff, D. W. Hutmacher, S. M. Cool. The stimulation of healing within a rat calvarial defect by mPCL-TCP/collagen scaffolds loaded with rhBMP-2. Biomaterials, vol. 30, no. 13, pp. 2479-2488, 2009.

[5] Q. J. Peng, X. M. Kang, T. T. Zhao. Effective virtual reality based building navigation using dynamic loading and path optimization. International Journal of Automation and Computing, vol. 6, no. 4, pp. 335-343, 2009.

[6] Y. Zhang, T. R. Zhou, X. H. Zhong. Optimization and simulation on filling path in FDM rapid prototyping process. Forging and Stamping Technology, vol. 33, no. 2, pp. 124127, 2008. (in Chinese)

[7] B. Asiabanpour, B. Khoshnevis. Machine path generation for the SIS process. Robotics and Computer-integrated Manufacturing, vol. 20, no. 3, pp. 167-175, 2004.

[8] G. Q. Jin, W. D. Li, L. Gao. An adaptive process planning approach of rapid prototyping and manufacturing. Robotics and Computer-integrated Manufacturing, vol. 29, no. 1, pp. 23-38, 2013.

[9] B. Asiabanpour, B. Khoshnevis. A new memory efficient tool path generation method for applying very large STL files in vector-by-vector rapid prototyping processes. In Proceedings of the 31st International Conference on Computers and Industrial Engineering, San Francisco, CA, USA, pp. 383-390, 2003.

[10] I. T. Ozbolat. Path planning for functionally graded materials in hollow tissue scaffold printing. In Proceedings of the ASME 2011 International Mechanical Engineering Congress and Exposition, IEEE, Denver, Colorado, USA, pp. 49-53, 2011.

[11] J. G. Sun. Computer Graphics, Beijing, China: Tsinghua University Press, pp. 357-476, 2009. (in Chinese)

[12] I. Zein, D. W. Hutmacher, K. C. Tan, S. H. Teoh. Fused deposition modeling of novel scaffold architectures for tissue engineering applications. Biomaterials, vol. 23, no. 4, pp. 1169-1185, 2002.

[13] H. Q. Tan. C++ Programming Design, Beijing, China: Tsinghua University Press, pp. 1-150, 2004. (in Chinese)

[14] Y. Zhang, X. L. Bai, Y. X. Liu. STEP-NC based high-level machining simulations integrated with CAD/CAPP/CAM. International Journal of Automation and Computing, vol. 9, no. 5, pp. 506-517, 2012.

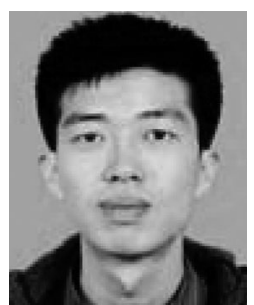

Wei-Hua Chen is a master student at Rapid Manufacturing Engineering Center of Shanghai University, China.

His research interests include rapid manufacturing and biological manufacturing equipment.

E-mail: chenwh518@gmail.com ORCID iD: 0000-0002-4707-9807 


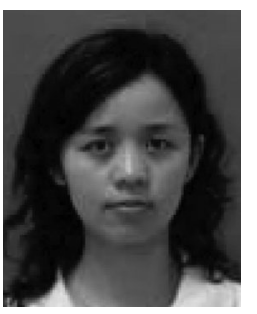

Yuan-Yuan Liu received her Ph. D. degree from Shanghai Jiao Tong University, China in 2008. Currently, she is an associate professor and deputy doctoral supervisor in Rapid Manufacturing Engineering Center at Shanghai University, China.

Her research interests include 3D printing technology and additive manufacturing equipment research.

E-mail: yuanyuan_liu@shu.edu.cn (Corresponding author) ORCID iD: 0000-0003-1568-0048

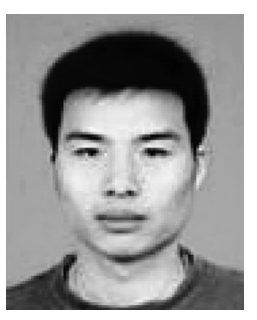

Fu-Hua Zhang is a master student at Rapid Manufacturing Engineering Center of Shanghai University, China.

His research interests include rapid manufacturing and biological manufacturing devices.

E-mail: zhangfuhua0506@gmail.com

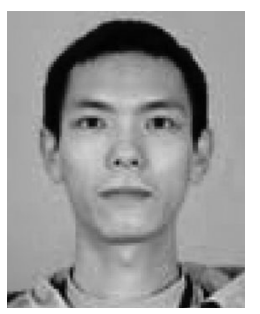

Yong-Ze Yu graduated from Tangshan College, China in 2007. He received the M. Sc. degree from Guangxi University of Science and Technology, China in 2010. He is currently a Ph. D. candidate at Shanghai University, China.

His research interests include the manufacturing of tissue engineering scaffolds by rapid prototyping and electrospinning.
E-mail: yuyz1023@sina.com

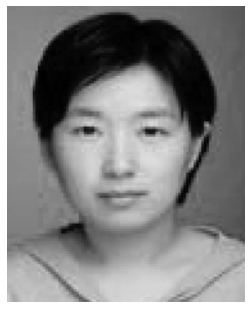

Hai-Ping Chen received the M. Sc. degree from Xinjiang University, China in 2010. She is currently a Ph. D. candidate at Shanghai University, China.

Her research interest is cell electrospinning.

E-mail: 519673062@qq.com

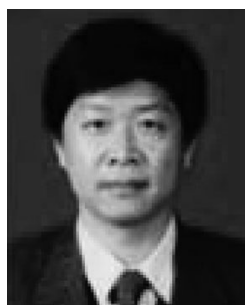

Qing-Xi Hu received his Ph. D. degree from Huazhong University of Science and Technology, China in 1997. Currently, he is a professor and doctoral supervisor at Rapid Manufacturing Engineering Center of Shanghai University, China. He is the director of Engineering Technical Training Center at Shanghai University, China.

He has been in charge of over 10 projects from National Natural Science Foundation, Key Projects of Science and Technology Commission and Municipal Commission of Shanghai. He has published 3 books, over 100 journal and conference papers and obtained more than 20 patents.

His research interests include 3D printing, biological manufacturing and contemporary integrated manufacture systems.

E-mail: huqingxi@shu.edu.cn 